EXTENDED REPORT

\title{
Correlation between surgical success rate and severity of congenital glaucoma
}

\author{
A Al-Hazmi, A Awad, J Zwaan, S A Al-Mesfer, I Al-Jadaan, A Al-Mohammed
}

Br J Ophthalmol 2005;89:449-453. doi: 10.1136/bjo.2004.047761

See end of article for authors' affiliations

Correspondence to:

Correspondence to:
Dr Abdulaziz Awad, King Khaled Eye Specialist Hospital, PO Box 7191, Riyadh 11 1462, Saudi Arabia; kkeshmeded@ awalnet.net.sa

Accepted for publication 28 August 2004

\begin{abstract}
Aim: To study the correlation between severity of primary congenital glaucoma (PCG) and success of three types of surgery.

Methods: This was a retrospective review of all records of patients diagnosed with PCG up to age 1 year who underwent goniotomy, trabeculotomy, or combined trabeculotomy-trabeculectomy with mitomycin C as initial procedure between 1982 and 2002 at the King Khaled Eye Specialist Hospital, Riyadh, Saudi Arabia. 532 paediatric glaucoma patients below age 1 year (820 eyes) with a minimum 1 year follow up were identified. The main outcome measures used for the surgeries were postoperative intraocular pressure, stability of the corneal diameter, and maintenance of corneal clarity. Surgical success was defined as a postoperative intraocular pressure of $\leqslant 21 \mathrm{~mm} \mathrm{Hg}$ without additional medical or surgical therapy, and with decreased corneal oedema, stabilised corneal diameter, and no additional optic nerve damage for at least 1 year after surgery. Complications, time of surgical failure, and follow up were recorded.

Results: The eyes were grouped into mild (249), moderate (342), and severe (229) PCG, based on intraocular pressure, corneal diameter, and clarity. All three surgical procedures resulted in high success rates of $81-100 \%$ for the mild form of PCG. Eyes classified with moderate glaucoma had a $13 \%, 40 \%$, and $80 \%$ success rate respectively for goniotomy, trabeculotomy, and combined trabeculotomy-trabeculectomy with mitomycin C. The success rate for severe PCG was $10 \%$ and $70 \%$ for trabeculotomy and combined surgery respectively. Goniotomy was never done for eyes with this condition.

Conclusion: Clinical classification of PCG is helpful for surgical decision making. The mild form has a high surgical success regardless of the procedure chosen. Combined trabeculotomy-trabeculectomy with mitomycin $\mathrm{C}$ gave the best results for moderate and severe cases of PCG.
\end{abstract}

$\mathrm{P}$ rimary congenital glaucoma (PCG) is a common ocular disorder in Saudi Arabia and is more severe than in Western countries. Treatment is surgical and is goniotomy, trabeculotomy, trabeculectomy, or combined trabeculotomy-trabeculectomy with (CTTM) or without (CTT) mitomycin C. The effectiveness of goniotomy or trabeculotomy in PCG is very high in Western countries with reported success rates of $81-90 \% .{ }^{12}$ This is in stark contrast with the low success rate in PCG patients in the Middle East. ${ }^{3}$ We undertook a retrospective study of three types of surgical procedures for PCG and examined the correlation between surgical success of these procedures and severity of the disease.

\section{METHODS}

Charts of patients diagnosed with PCG (from birth to I year of age) who underwent goniotomy, trabeculotomy, or CTTM as an initial procedure from 1982 to 2002 at the King Khaled Eye Specialist Hospital (KKESH) in Riyadh, Saudi Arabia, were retrospectively reviewed. Patients older than 1 year, with follow up of less than 1 year, history of previous surgery outside our hospital, and congenital glaucoma associated with ocular anomalies and/or systemic diseases were excluded. Patients who had trabeculectomy performed on them were also excluded because they were very few in number, and it was usually a secondary procedure. An additional 20 patients were excluded because their Schlemm's canal could not be identified.

Demographic data were documented. Patients had complete ophthalmic examinations while under general anaesthesia before surgery. These included biomicroscopy, measurement of intraocular pressure (IOP), and corneal diameter. The IOP was measured by pneumotonometer and Tonopen; both tools were used under general anaesthesia or under sedation. Anterior chamber and corneal clarity were recorded. Fundus examination by direct and indirect ophthalmoscopy was performed depending on clarity of the media. Refraction was done infrequently because most patients had poor media because of corneal oedema and as cycloplegic agents were not routinely instilled before operations. Eyes were classified based on IOP, corneal diameter and corneal clarity. Each of these parameters was given a score from 1 to 3 . The total score determined classification as mild (1-3), moderate (4-6), or severe (7-9) PCG (table 1).

All surgeries were performed by experienced surgeons with minor variations. Surgical techniques for goniotomy and trabeculotomy were based on those of Barkan ${ }^{4}$ and McPherson and Berry, ${ }^{2}$ respectively. Our variation of the technique for CTTM was reported previously. ${ }^{5}$

We recorded the type of glaucoma surgery, intraoperative and postoperative complications and their management. Postoperative follow ups (minimum 1 year and maximum 2 years) were documented. Most cases were seen at the end of the first week or more often as necessary, and thereafter at $1,3,6,9$ months, and at 1 and 2 years. At each visit, patients were fully evaluated, usually under sedation with chloral hydrate. Retinoscopy was performed when the cornea was sufficiently clear.

Abbreviations: CTTM, combined trabeculotomy-trabeculectomy with mitomycin $\mathrm{C} ; \mathrm{CTT}$, combined trabeculotomy-trabeculectomy without mitomycin C; IOP, intraocular pressure; PCG, primary congenital glaucoma 
Table 1 Classification used for primary congenital glaucoma

\begin{tabular}{llll}
\hline Severity & IOP $(\mathbf{m m ~ H g})$ & $\begin{array}{l}\text { Corneal diameter } \\
(\mathbf{m m})\end{array}$ & Corneal clarity \\
\hline Mild & $<25$ & $<13$ & Good \\
Moderate & $25-35$ & $13-14.5$ & Fair \\
Severe & $>35$ & $>14.5$ & Poor \\
\hline
\end{tabular}

Surgical success was defined as a postoperative IOP of $\leqslant 21 \mathrm{~mm} \mathrm{Hg}$ without additional medical or surgical therapy, and with decreased corneal oedema, stabilised corneal diameter, and stability of the optic nerve for at least 1 year after surgery.

The study protocol was approved by KKESH's research council and human ethics committee/institutional review board.

\section{RESULTS}

Analysis included 820 eyes of 532 patients. Ninety six per cent of the patients were local Saudi Arabians. Table 2 gives demographic data on the patients for each of the three surgeries performed. Trabeculotomy was the most common procedure done in $418(51 \%)$ eyes.

A family history positive for PCG was present in 109 patients $(21 \%)$ and 323 patients $(61 \%)$ were the products of consanguineous marriages. These patients had more severe presentations and did require more surgeries to control the disease. Four hundred and ten patients (77\%) were bilaterally affected.

Two hundred and forty nine (30\%) eyes met our criteria for mild PCG, 342 (42\%) eyes were classified as moderate PCG, and $229(28 \%)$ eyes as severe. The age of presentation for each group is shown in table 2. Severe cases had more severe disease (for example, anomalous angle) with high IOP, leading to more buphthalmos.

Our definition of surgical success was used to compile table 3, which shows success rates in different time periods.
The first period, 1982 to 1990 , before the introduction of CTTM, showed high failure rates. In the following two periods, 1991-4 and 1995-2002, failure rates decreased, especially in the latter period when significant experiences with antimetabolites and CTTM procedure were gained. Overall surgical success rates (goniotomy $52 \%$, trabeculotomy $41 \%$, CTTM 75\%) were low compared to results from Western countries. Table 4 , in which the eyes were divided into mild, moderate, and severe cases of PCG, shows mild cases did well after goniotomy or trabeculotomy, unlike moderate cases. While severe cases did poorly after trabeculotomy, a 75\% success rate was obtained with CTTM.

Mild cases of PCG treated with goniotomy showed only a modest failure rate after 2 years. In contrast, in about two thirds of the patients with moderate PCG, the IOP was no longer in control at 2 years (fig 1). Moderate and severe PCG showed increasing failure with time after surgery, when treated with trabeculotomy. At the end of 2 years, only $10 \%$ of severe cases were controlled (fig 2). After CTTM, IOP control was much better maintained although severe PCG showed less success than the mild and moderate form (fig 3 ).

Intraoperative and postoperative complications for all three surgeries (table 5 ) ranged from hyphaema $>50 \%$ ( 17 cases) to exudative retinal detachment (one case). Hyphaema occurred in all three surgeries; all cases resolved spontaneously except for one case in the goniotomy group which required anterior chamber washout. Choroidal detachment, the second most frequent complication, only occurred in the trabeculotomy group (five cases) and the combined group

Table 2 Demographic data on primary congenital glaucoma cases obtained from KKESH records from 1982 to 2002

\begin{tabular}{|c|c|c|c|c|}
\hline Data & $\begin{array}{l}\text { Total } \\
\text { (\%) }\end{array}$ & $\begin{array}{l}\text { Goniotomy } \\
\text { No (\%) }\end{array}$ & $\begin{array}{l}\text { Trabeculotomy } \\
\text { No (\%) }\end{array}$ & $\begin{array}{l}\text { CTTM }^{*} \\
\text { No }(\%)\end{array}$ \\
\hline Patients & 532 & $155(29)$ & $292(55)$ & $85(16)$ \\
\hline Eyes & 820 & $254(31)$ & $418(51)$ & $148(18)$ \\
\hline \multicolumn{5}{|l|}{ Sex } \\
\hline Male & $434(53)$ & $136(54)$ & $220(53)$ & 78 (53) \\
\hline Female & $386(47)$ & $118(46)$ & $198(47)$ & $70(47)$ \\
\hline \multicolumn{5}{|c|}{ Age (months) } \\
\hline Mean & 4.2 & 4.8 & 4.2 & 3.5 \\
\hline Range & $0.1-12$ & $0.4-12$ & $0.3-12$ & $0.1-12$ \\
\hline \multicolumn{5}{|c|}{ Follow up (months) } \\
\hline Mean & 15 & 15 & 14 & 16 \\
\hline Range & $12-24$ & $12-24$ & $12-24$ & $12-24$ \\
\hline
\end{tabular}

Table 3 Success rates of three types of surgery for primary congenital glaucoma over time

\begin{tabular}{|c|c|c|c|c|c|c|}
\hline \multirow[b]{2}{*}{ Period } & \multicolumn{2}{|c|}{ Goniotomy } & \multicolumn{2}{|c|}{ Trabeculotomy } & \multicolumn{2}{|c|}{ СТTM* } \\
\hline & No & Success (\%) & No & Success (\%) & No & Success (\%) \\
\hline $\begin{array}{l}1982-90 \\
1991-4 \\
1995-2002 \\
\text { Total }\end{array}$ & $\begin{array}{r}170 \\
59 \\
25 \\
254\end{array}$ & $\begin{array}{r}71(42) \\
40(67) \\
20(80) \\
131(52)\end{array}$ & $\begin{array}{r}236 \\
129 \\
53 \\
418\end{array}$ & $\begin{array}{r}68(29) \\
61(47) \\
44(82) \\
173(41)\end{array}$ & $\begin{array}{r}29 \\
119 \\
148\end{array}$ & $\begin{array}{r}25(86) \\
86(72) \\
111(75)\end{array}$ \\
\hline
\end{tabular}


Table 4 Outcome of three surgical procedures for primary congenital glaucoma in 820 eyes

\begin{tabular}{|c|c|c|c|c|c|c|c|c|c|}
\hline \multirow[b]{2}{*}{ Severity } & \multicolumn{3}{|c|}{ Goniotomy } & \multicolumn{3}{|c|}{ Trabeculotomy } & \multicolumn{3}{|c|}{ СтाM* } \\
\hline & No & Success (\%) & Failure (\%) & No & Success (\%) & Failure (\%) & No & Success $(\%)$ & Failure (\%) \\
\hline Mild & 145 & $117(81)$ & $28(19)$ & 102 & $93(90)$ & $10(10)$ & 2 & $2(100)$ & $0(0)$ \\
\hline Moderate & 109 & 14 (13) & 95 (87) & 163 & $65(40)$ & $98(60)$ & 70 & $56(80)$ & $14(20)$ \\
\hline Severe & 0 & 0 & 0 & 153 & $15(10)$ & $138(85)$ & 76 & $53(70)$ & $23(30)$ \\
\hline Total & 254 & 131 (52) & $123(48)$ & 418 & $173(41)$ & 246 (59) & 148 & 111 (75) & $37(25)$ \\
\hline
\end{tabular}

(six cases). All cases resolved spontaneously except for one after CTTM which required drainage of the effusion. There were 10 complications of shallow anterior chamber with one case in the goniotomy group, three in the trabeculotomy group, and six in the combined group. Eight reformed spontaneously but two of the eyes after CTTM needed anterior chamber reformation. Cataract was also seen in all three groups: the goniotomy group had five cases, two of which required lens aspiration; the trabeculotomy group had three cases, one of which required lens aspiration; and the combined surgery group had one case which did not require surgical intervention. In total, complications were most common when the CTTM technique was undertaken (31/73 cases).

Antiglaucoma medications were used at times to control IOP after failed surgery in about $35 \%$ of the cases. Additional surgeries were performed for eyes uncontrolled with antiglaucoma therapy. Many eyes required more than one procedure, either a repetition of the surgery or, if that failed, procedures such as implantation of an Ahmed valve or cyclodestructive therapy.

\section{DISCUSSION}

The majority of familial cases of PCG in Saudi Arabia are inherited as an autosomal recessive trait ${ }^{6}$ and linked to locus GLC3A on chromosome 2p21. ${ }^{7}$ This locus has been further identified as CYPIBl, the gene responsible for the production of a cytochrome P4501Bl. Owing to the frequency of consanguineous marriages in the population, ${ }^{8}$ PCG is common. Even at birth, both corneas are usually substantially enlarged, cloudy, and oedematous. ${ }^{910}$ Indeed, 582 of 820 eyes $(71 \%)$ in this study had corneal opacities and the mean preoperative corneal diameter was $15 \mathrm{~mm}$. Thus, the

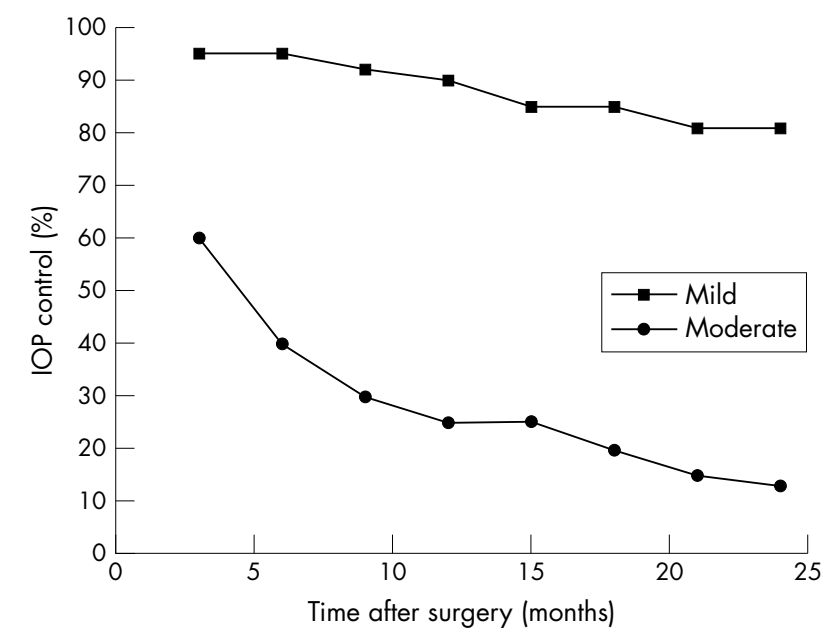

Figure 1 Goniotomy resulted in excellent control of intraocular pressure over a 2 year period in primary glaucoma cases classified as mild (squares). In moderate cases the success rate dropped significantly over time (circles). disease is more serious than usually seen in Western countries, possibly because of the presence of genetic or environmental modifiers of the expression of CYPIBl in the Saudi Arabian population.

Hoskins et al ${ }^{11}$ proposed an anatomical classification based on gonioscopy and divided PCG into three groups: trabeculodysgenesis, iridotrabeculodysgenesis, and corneotrabeculodysgenesis. Luntz, ${ }^{12}$ who was the first to propose CTT, ${ }^{13}$ reported the success of goniotomy and trabeculotomy was $90 \%$ for trabeculodysgenesis, but only $30 \%$ for iridotrabeculodysgenesis and corneotrabeculodysgenesis. He recommended goniotomy and trabeculotomy for the first group but a combination of trabeculotomy and trabeculectomy for the latter two groups.

Hoskins's classification is applicable only to cases of clear cornea. The majority of our study group had severe corneal oedema which did not permit a view of the angle by gonioscopy. Therefore, we used a classification system designed mainly for advanced cases of PCG. The 820 eyes with PCG were grouped into mild, moderate, and severe cases based on IOP, corneal diameter, and corneal integrity or clarity.

The prognosis of PCG is affected by various factors such as time of presentation, severity, and time of surgery. The earlier in life the disease occurs the worse the prognosis, ${ }^{14}$ and the more severe the case the higher the failure rate. While medical treatment of PCG is useful as a temporary measure or as adjunctive therapy, surgery is always necessary. ${ }^{15}$ It has been shown the majority of failed surgeries occur within the first year. ${ }^{16}$

Controlling IOP is the major problem of PCG. Prolonged high IOP can cause irreversible optic nerve damage, corneal opacity, staphyloma, refractive error, amblyopia, and may lead to loss of vision. Timely reduction of IOP and

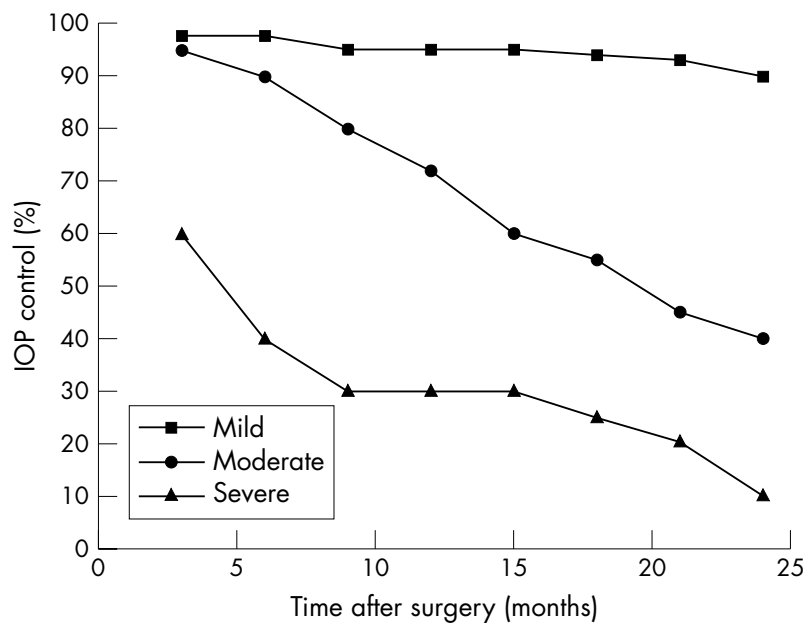

Figure 2 Mild primary congenital glaucoma (squares) did well after trabeculotomy during 2 years of follow up. Moderate (circles) and severe cases (triangles) showed an increasing failure rate over time. 


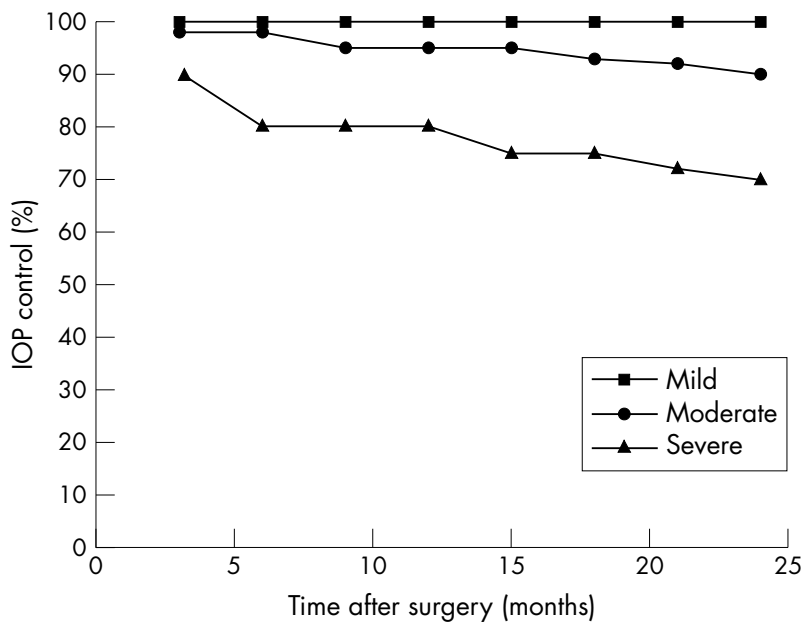

Figure 3 With combined trabeculotomy-trabeculectomy with mitomycin C, intraocular pressure control was maintained well in 100\% of mild (squares), $80 \%$ of moderate (circles), and $70 \%$ of severe PCG (triangles) over 2 years.

stabilisation of refractive error are essential to avoid the sequelae.

There is a great disparity between the results obtained in the early years after the opening of KKESH in 1982 and the more recent period. During the first 8 years (1982 to 1990), PCG cases were treated by goniotomy or trabeculotomy with success rates of $42 \%$ and $29 \%$ respectively. Success rates steadily increased after 1991 with the introduction of mitomycin C and the debut of CTTM at KKESH in 1994. From 1995 to 2002, the success rate improved to $80 \%$ for goniotomy, to $82 \%$ for trabeculotomy, and to $72 \%$ for CTTM. These improved results are probably the result of more extensive primary healthcare facilities within the kingdom, resulting in earlier referrals, better equipment availability, and perhaps surgeons being more selective in choice of procedure relative to degree of seriousness of PCG. The disparity of surgical results between 1982 and 2002 led us to develop a classification to correlate severity of PCG with clinical findings that would assist in selecting an optimal surgical approach to obtain IOP control in PCG patients.

Successful control of IOP after goniotomy has been reported to vary from $81-94 \%$ with one or multiple procedures in the Western world. ${ }^{12}{ }^{17}$ This result is probably due to the general mildness of PCG which is the norm in populations in developed countries. Goniotomy as the initial procedure was performed in our institution in 254 eyes with a success rate of $81 \%$ over a 2 year follow up period when PCG was mild. It dropped dramatically to $13 \%$ for the moderate state. The procedure was not attempted for severe cases of
PCG. In the early years at KKESH, goniotomies were performed even if the cornea was moderately opaque. Patients were admitted a few days before surgery and managed with extensive medical antiglaucoma therapy. Topical sodium chloride was added to reduce the corneal oedema to improve visualisation for goniotomy. Records indicate that most of these cases failed within l week to 2 months postoperatively, and additional surgeries were needed.

Trabeculectomy is a straightforward procedure with generally satisfactory results for congenital glaucoma when combined with mitomycin C. ${ }^{18}{ }^{19}$ Our previous study showed that CTTM had higher success rates than trabeculectomy alone. ${ }^{19}$ However, we omitted trabeculectomy from the present study because the procedure was undertaken only occasionally as primary surgery in the age group below 1 year.

Trabeculotomy was undertaken as the initial procedure in 418 eyes with a success rate of $90 \%$ for mild PCG, $40 \%$ for moderate, and $10 \%$ for severe glaucoma. These figures strongly suggest trabeculotomy is an appropriate choice only for mild cases of PCG in our population. It has been reported that Schlemm's canal cannot be located in $11 \%$ to $15 \%$ of cases. ${ }^{20}{ }^{21}$ In our study the canal was not identified in 20 (5\%) of 418 trabeculotomy cases. We surmise that with advanced glaucoma and subsequent growth of the cornea, the topographical anatomy of the structures around the chamber angle changes, causing shifts in the position of Schlemm's canal. Failure of initially successful trabeculotomy may occur, when meshwork tissues gradually overgrow the trabeculotomy site or synechiae form. Beck and Lynch described a technique of performing $360^{\circ}$ trabeculotomy in a single procedure, using a 6-0 proline suture threaded through Schlemm's canal under visualisation by gonioscopy with a reported $87 \%$ success rate. ${ }^{22}$ They commented that among its advantages were avoidance of difficulties sometimes encountered with a metal probe and preservation of the conjunctiva. However, the technique is not possible for severe cases of PCG because corneal opacification would not permit visualisation by gonioscopy.

The combined trabeculotomy-trabeculectomy procedure has been described with and without the use of mitomycin C. ${ }^{5} 161819$ 23-25 Its advantage is that it does not require corneal clarity; it allows the trabeculectomy to be placed correctly, as Schlemm's canal has to be located, and provides additional filtering surgery. Failure to identify the canal does not lead to total failure of the surgery as there is another drainage pathway. Mitomycin C increases success by suppressing fibrosis and vascular ingrowth. ${ }^{25}$ Mullaney et al ${ }^{5}$ recently reported a $78 \%$ success rate for combined trabeculotomy and trabeculectomy for PCG: most of them with mitomycin C. This is similar to our result of a 75\% success rate for all CTTM procedures done at KKESH. Mandal et $a^{23}(\mathrm{n}=182)$ and

Table 5 Intraoperative and postoperative complications of primary congenital glaucoma

\begin{tabular}{lllll}
\hline Complication & Total & Goniotomy & Trabeculotomy & CTTM* \\
\hline Hyphaema $>50 \%$ & 17 & 7 & 6 & 4 \\
Choroidal detachment & 11 & 0 & 5 & 6 \\
Shallow anterior chamber & 10 & 1 & 3 & 6 \\
Cataract & 9 & 5 & 3 & 1 \\
Iridodialysis & 8 & 3 & 4 & 2 \\
Descemet detachment & 5 & 2 & 1 & 4 \\
Hypotony & 5 & 1 & 0 & 3 \\
Vitreous loss & 4 & 0 & 1 & 1 \\
Blebitis & 2 & 0 & 0 & 1 \\
Endophthalmitis & 1 & 0 & 0 & 1 \\
Exudative retinal detachment & 1 & 0 & 0 & \\
& ${ }^{*}$ CTTM= combined trabeculectomy-trabeculectomy with mitomycin C. & & \\
\end{tabular}


$\operatorname{Elder}^{24}(\mathrm{n}=16)$ reported success rates of combined trabeculotomy and trabeculectomy to be approximately $94 \%$. In our previous study, ${ }^{19}$ the addition of mitomycin $\mathrm{C}$ to the combined procedure gave IOP control in $70 \%$ of paediatric glaucoma patients with a follow up of $1-5$ years. However, success decreased as the age decreased $(50 \%$ in patients younger than 6 months). Mitomycin $\mathrm{C}$ augmentation of the combined procedure increases the surgical success rate with relatively few complications in paediatric glaucoma surgery. ${ }^{15} 181925$ However, our data indicate that more complications occurred in the CTTM group, especially hypotony, vitreous loss, blebitis, and exudative retinal detachment. The incidence of complications may be attributable to age: the average age in our study groups is 4.2 months. In spite of these complications, CTTM has been the procedure of choice for moderate and severe forms of PCG during the last 5 years at KKESH.

In summary, a classification of PCG in mild, moderate, or severe forms, based on IOP, corneal diameter, and corneal integrity can be helpful for surgical decision making. The mild form of congenital glaucoma generally responds successfully to surgery, no matter which procedure is chosen. Combined trabeculotomy-trabeculectomy with adjunctive mitomycin $\mathrm{C}$ results in better IOP control in the moderate and severe forms.

\section{Authors' affiliations}

A Al-Hazmi, A Awad, J Zwaan, S A Al-Mesfer, Pediatric Ophthalmology Division, King Khaled Eye Specialist Hospital, Riyadh, Saudi Arabia

A Al-Hazmi, Department of Ophthalmology, King Fahd Central Hospital, Jizan, Saudi Arabia

I Al-Jadaan, A Al-Mohammed, Glaucoma Division, King Khaled Eye Specialist Hospital, Riyadh, Saudi Arabia

J Zwaan, Department of Ophthalmology, University of Texas Health Sciences Center, San Antonio, TX, USA

Presented in part as a poster at the Joint 103rd Annual Meeting of the American Academy of Ophthalmology and XXII Congress of the PanAmerican Association of Ophthalmology, 24-27 October 1999, Orlando, FL, USA.

\section{REFERENCES}

1 Anderson DR. Trabeculotomy compared to goniotomy for glaucoma in children. Ophthalmology 1983;90:805-6.
2 McPherson SD Jr, Berry DP. Goniotomy vs external trabeculotomy for developmental glaucoma. Am J Ophthlamol 1983;95:427-31.

3 Debnath SC, Teichmann KD, Salamah K. Trabeculectomy versus trabeculotomy in congenital glaucoma. Br J Ophthalmol 1989;73:608-11.

4 Barkan O. Technique of goniotomy. Arch Ophthalmol 1938;19:217-21.

5 Mullaney PB, Selleck C, Al-Awad A, et al. Combined trabeculotomy and trabeculectomy as an initial procedure in uncomplicated congenital glaucoma. Arch Ophthalmol 1999;117:457-60.

6 Francois J. Congenital glaucoma and its inheritance. Ophthalmologica 1980;181:61-73.

7 Sarfarazi M, Akarsu AN, Hossain A, et al. Assignment of a locus (GLC3A) for primary congenital glaucoma (buphthalmos) to $2 \mathrm{p} 21$ and evidence for genetic heterogeneity. Genomics 1995;30:171-7.

8 Bejiani BA, Lewis RA, Tomey KF, et al. Mutations in CYP1B1, the gene for cytochrome P4501B1, are the predominant cause of primary congenital glaucoma in Saudi Arabia. Am J Hum Gent 1998:62:325-33.

9 Jaafar MS. Care of infantile glaucoma patient. In: Reinecke RD, ed. Ophthalmology annual, Vol 7. New York: Raven Press, 1988:15-37.

10 Tabbara KF, Badr IA. Changing pattern of childhood blindness in Saudi Arabia. Br J Ophthalmol 1985:69:312-5.

11 Hoskins HD Jr, Shaffer RN, Hetherington J. Anatomical classification of the developmental glaucomas. Arch Ophthalmol 1984;102:1331-6.

12 Luntz MH. The choice of surgical procedure in congenital, infantile, and juvenile glaucoma. J Ophthalmic Nurs Technol 1990:9:100-1.

13 Luntz MH. Congenital, infantile, and juvenile glaucoma. Ophthalmology 1979;86:793-801.

14 Dureau $\mathbf{P}$, Dollfus H, Cassegrain C, et al. Long-term results of trabeculectomy for congenital glaucoma. J Pediatr Ophthalmol Strabismus 1998:35:198-202.

15 Turach ME, Aktan G, Idil A. Medical and surgical aspects of congenital glaucoma. Acta Ophthalmol (Scand) 1995;73:261-3.

16 Mandal AK, Walton DS, John T, et al. Mitomycin C-augmented trabeculectomy in refractory congenital glaucoma. Ophthalmology 1997;104:996-1001.

17 Shaffer RN. Prognosis of goniotomy in primary infantile glaucoma (trabeculodysgenesis). Trans Am Ophthalmol Soc 1982;80:321-5.

18 Agarwal HC, Sood NN, Sihota R, et al. Mitomycin-C in congenital glaucoma. Ophthalmic Surg Lasers 1997;28:979-85.

19 Al-Hazmi A, Zwaan J, Awad A, et al. Effectiveness and complications of mitomycin $C$ use during pediatric glaucoma surgery. Ophthalmology 1998;105:1915-20.

20 Elder MJ. Congenital glaucoma in the West Bank and Gaza Strip. Br J Ophthalmol 1993;77:413-6.

21 Harms H, Dannheim R. Epicritical consideration for 300 cases of trabeculotomy "ab externo". Trans Ophthalmol Soc UK 1969:89:491-9.

22 Beck AD, Lynch MG. $360^{\circ}$ trabeculotomy for primary congenital glaucoma. Arch Ophthalmol 1995;113:1200-2.

23 Mandal AK, Naduvilath TJ, Jayagandan A. Surgical results of combined trabeculotomy-trabeculectomy for developmental glaucoma. Ophthalmology 1998; 105:974-82.

24 Elder MJ. Combined trabeculotomy-trabeculectomy compared with primary trabeculectomy for congenital glaucoma. $\mathrm{Br} J$ Ophthalmo 1994:78:745-8.

25 Susanna R Jr, Oltrogge EW, Carani JCE, et al. Mitomycin as adjunct chemotherapy with trabeculectomy in congenital and developmental glaucomas. J Glaucoma 1995;4:151-7. 